\title{
Rideaux, pots de fleurs et escaliers modern style
}

Aménagement des espaces et fonction des objets dans El baile, d'Edgar Neville

\section{Alfonso San Miguel Montes}

\section{(2) OpenEdition}

1 Journals

\section{Édition électronique}

URL : http://journals.openedition.org/agedor/4298

DOI : 10.4000 /agedor.4298

ISSN : 2104-3353

Éditeur

Laboratoire LISAA

\section{Référence électronique}

Alfonso San Miguel Montes, «Rideaux, pots de fleurs et escaliers modern style », L'Âge d'or [En ligne], 1 । 2008, mis en ligne le 10 juin 2008, consulté le 15 juin 2020. URL : http://journals.openedition.org/ agedor/4298; DOI : https://doi.org/10.4000/agedor.4298

Ce document a été généré automatiquement le 15 juin 2020.

L'Âge d'or. Images dans le monde ibérique et ibéricoaméricain 


\title{
Rideaux, pots de fleurs et escaliers modern style
}

Aménagement des espaces et fonction des objets dans El baile, d'Edgar Neville

\author{
Alfonso San Miguel Montes
}

\section{Introduction}

1 Je voudrais citer tout d'abord ces propos d'Émile Gallé, figure clé dans l'appréhension de l'Art nouveau français (mais aussi du Modern style, Jugendstil ou encore Modernisme $)^{1}$. Ils ont été écrits en 1900, à une époque où la parenté entre l'Art Nouveau et le cinéma était déjà clairement ressentie :

À ce rendez-vous des intelligences créatrices, l'avenir retrouvera, dans le mobilier contemporain comme dans les autres arts du décor, non pas l'anarchie, mais, en la même ambiance vitale, un meuble de caractères généraux modernes, sous ces traits de famille heureuse : la personnalité, l'individualité, la réflexion, le souci de la logique, l'observation de la nature, l'émotion, l'imagination, l'enthousiasme, la sincérité, la vie. ${ }^{2}$

2 Ils m'ont semblé importants, dans la mesure où quelques-uns des principes évoqués animent les décors choisis pour El baile, l'avant-dernier film réalisé par Edgar Neville, en 1959. Dans ce cas, littérature et filmicité n'ont jamais été si proches puisqu'il s'agit d'une pièce écrite par Neville et portée sept ans plus tard à l'écran. Le récit nevillien prend corps dans un contexte résolument urbain, un Madrid joyeux et mirobolant, tout prêt à savourer les joies de la Belle époque.

Or, si dans un premier temps Madrid se caractérise par ses signes extérieurs, ceux-ci ne prendront véritablement du sens que par rapport aux espaces intérieurs où le film se déroule majoritairement. C'est sur ce plan que les doctrines du Lorrain s'adaptent à ce sujet. Les réponses esthétiques que donne l'Art Nouveau peuvent ainsi être une précieuse aide pour expliquer le sens symbolique et émotionnel attribué aux objets et à l'aménagement de l'espace dans le film. Neville place ses créatures, un trinôme particulier composé de Pedro, de son épouse Adela et de Julián, ami du couple mais 
amoureux depuis sa jeunesse de la jeune femme, à l'intérieur d'un hôtel particulier d'aspect opulent et raffiné. Tous les trois semblent y habiter en parfaite et étrange symbiose. Cette situation, qui met en scène un univers sentimental inouï où la compréhension et le respect l'emportent sur la pulsion sexuelle et la rivalité amoureuse pouvant exister entre les deux hommes, permet au réalisateur de mener une réflexion sur la recherche de l'amour éternel. En réalité, l'approche triangulaire répond plutôt au dédoublement d'une même personnalité masculine. D’un côté Julián, l'éternel amoureux, à la fois jaloux maladif et protecteur, de l'autre Pedro, mari-amant affectueux mais quelquefois inattentif aux besoins de son épouse. Ce qui les relie est la même dévotion partagée par les deux hommes pour Adela, le troisième sommet du triangle. Elle condense en elle-même toutes les caractéristiques du modèle de femme nevillienne et c'est autour d'elle que tournent les différentes situations développées dans le film³ .

Édifiant un univers en « huis clos » à l'image des personnages « sous les traits de famille heureuse ", tel que le signalait Gallé, les formes construites aux allures modern style et dotées d'une signification spécifique dans le profilmique sont assez loquaces. Elles parlent tout d'abord de personnalité et d'individualité, celles d'Adela qui bâtit un microcosme domestique en résonance avec son goût et ses désirs, peuplé de guéridons de style, de nymphes et de buffets sculptés aux lignes sinueuses, rappelant des organismes vivants. La jeune femme symbolise l'éternel féminin, la beauté expressive, comme elle existe dans la nature et dans la vie. Le contre-pied à la beauté des meubles et des objets modernistes qui composent l'univers féminin, celui attribué à Adela, sera «le souci de la logique » et "l'observation de la nature », personnifiés par Pedro et Julián, entomologistes passionnés de natures mortes et naturalisées. L'interprétation de la nature est fréquemment conseillée comme la seule source d'une saine invention. Dans le film, elle aboutit à une abstraction sur deux plans : d'une part la fascination pour les formes organiques rendues presque vivantes et visibles dans les stylisations curvilignes et végétales présentes dans les meubles, les boiseries des fenêtres, la balustrade de l'escalier; d'autre part, l'intérêt scientifique porté par les deux amis au domaine des sciences naturelles à travers l'étude des insectes naturalisés, des êtres jadis vivants, objets d'analyse dépouillés de mobilité dans le temps de la fiction. L'harmonie dans laquelle baignent ces trois personnages ne serait possible si, dans leurs rapports réciproques, il n'y avait l'inventivité et l'amour de la nature vécus avec « l'émotion, l'imagination, l'enthousiasme, la sincérité » dont parlait Gallé. Sentiments intimement liés aux rapports que tous les trois, à leur façon, entretiennent avec les objets rassemblés dans leur demeure.

Dans la conception de la mise en scène de son film, Neville s'en fait ainsi l'écho en établissant un parallèle entre le reflet dynamique afférent à la « vie » - ou " non-vie » des objets, dans une tentative de fixer leur «âme dans la surface et dans l'espace » et les inquiétudes spirituelles qui animent la vie de Pedro, de Julián et de leur Adela adorée dont ils essayent, en quête d'éternité, de préserver la vie et la beauté à travers le temps.

6 Car El baile est aussi un film qui parle du temps. La temporalité devient un scalpel narratif qui instrumentalise les comportements des personnages. De par son origine théâtrale, dont Neville a bien voulu garder les traces dans la structure en trois actes, le récit est construit sur la base d'une temporalité fracturée en trois temps, avec deux ellipses de vingt-cinq ans chacune. Il dépeint un triptyque des âges de l'homme: la 
jeunesse, l'âge mûr et la sénescence. Dans la pièce de théâtre, elles trouvent leur correspondance dans le changement d'acte et sont, d'un point de vue technique, brillamment réussies dans le film. Les rapports espace-temps acquièrent toute leur dimension sémantique.

\section{Acte I. Fixation du récit dans un cadre spatio-temporel}

7 Au début du film, Neville organise l'espace en acheminant graduellement le spectateur vers l'espace scénique où le récit prendra véritablement corps, l'intérieur de la résidence. Il place progressivement les personnages au cœur du dispositif représentatif. Il dévoile sa construction spatiale comme une structuration vertébrée qui se met en place dès la première séquence.

8 Afin de guider idéalement le spectateur dans sa quête de cohérence effective au sein de l'espace architectural, il passe du plus général au plus particulier, en allant de l'extérieur à l'intérieur et, en ce qui concerne les plans, du plus large au plus serré. En outre, le réalisateur ajoute quelques signes précis qui permettent de le situer dans un cadre temporel facilement reconnaissable, le Madrid du début du XX $\mathrm{XX}^{\mathrm{e}}$ siècle, avec les véhicules anciens et les tenues vestimentaires propres à l'époque.

Le film s'ouvre sur de très beaux plans d'ensemble du parc du Retiro où Adela et Julián, accomplissant un rituel courant et commun à la bonne société madrilène de l'époque, se promènent en voiture. Le plan qui suit est aussi bref que signifiant. Dans ce Madrid tonitruant, où le moderne a toujours su côtoyer la tradition, Neville choisit de s'attarder sur un immeuble remarquable, le Palais de Longoria, de l'intérieur duquel sortent Julián et Adela après avoir acheté quelques chapeaux pour cette dernière. Le fait de montrer cet édifice a deux fonctions : la première, figer le récit dans un cadre spatio-temporel visible; la seconde, bien plus subtile, en guise de cataphore cherchant une redondance du propos plastique, anticiper les mêmes éléments constructifs qui viennent définir l'espace architectural dans lequel se déroulera le reste du récit et qui sera montré aussitôt après. Il s'agit d'une mise en scène visuellement surchargée, formellement théâtrale, volontairement choisie par Neville qui jamais n'occulte la pièce à l'origine de ce film dont il est l'auteur. Cet hôtel moderniste d'une très grande beauté de lignes est en réalité une interprétation érudite d'un mouvement culturel auquel Grases Riera, son architecte, était parfaitement étranger ${ }^{4}$. Il a imité ses éléments les plus signifiants, surtout sur les façades extérieures, d'un indéniable expressionnisme, qui font office de bouleversante toile de fond. Neville se réapproprie cette architecture, un espace d'imitation à la base, pour l'utiliser comme une sorte de scénographie baroque qui s'adapte parfaitement aux architectures des intérieurs dont le sens reste essentiel dans la construction dramatique de l'œuvre.

Adela et Julián arrivent à la maison. La caméra découvre l'espace de l'action. Le réalisateur situe la première scène dans un cadre qui reproduit fidèlement les indications données par les didascalies de la pièce de théâtre : «Living room 1900. Des rideaux, des pots de fleurs et des ornements Modern style ». On s'aperçoit d'emblée que l'ornement représente effectivement quelque chose de plus qu'un simple décor. Outre l'expression d'une volonté de fabriquer une ambiance distinguée et cosmopolite, il devient l'expression d'une fonctionnalité sublimée. Il est avéré que, dans les objets Art Nouveau, le formel et le fonctionnel entrent en symbiose. Les lignes aérées, les sinuosités des formes recherchant l'expression d'un mouvement fluide, résultat d'une 
stylisation qui renvoie très souvent au monde floral, font écho à l'univers symbolique. Dans $\mathrm{El}$ baile, le décor réussit à sortir de son rôle souvent secondaire dans la mesure où il donne davantage de profondeur à l'étude des personnages.

11 Dès qu'on franchit la porte d'entrée, on pénètre dans les domaines d'Adela. La jeune femme arpente la grande pièce en déposant sur les différents meubles les paquets avec les achats qu'elle vient d'effectuer en compagnie de son ami, dévoilant, au gré de son parcours, cet espace - son espace - bâti sous le signe de la beauté, de l'harmonie et de la féminité. Véritable "marquage de territoire». Lorsque Julián s'apprête à déposer quelques boîtes à chapeaux sur un guéridon, Adela s'exclame: "Ne pose pas cela làdessus. Ça peut casser le guéridon $»^{5}$. Même si «Les objets ne doivent pas seulement être utiles, mais donner à reconnaître nettement vers l'extérieur leur aptitude à être utiles $^{6} "$, pour Adela, un guéridon de style restera un guéridon de style, exposant la prééminence de sa fonction plastique sur l'aspect pratique. Adela, quant à elle, saura rester une femme, quelles que soient les circonstances, et afficher avec la même intensité sa coquetterie, et ce, malgré le temps fuyant qui emportera sa beauté vingtcinq ans plus tard, tout comme ses illusions. Chaque objet joue le rôle d'indice qui exhibe les traits de sa personnalité : les fauteuils où elle s'installe, son guéridon préféré et l'escalier moderniste (lieu obligé de passage entre espaces symboliques, qu'elle gravit et descend, circulant librement dans un espace qu'elle a apprivoisé, pour montrer à son mari sa dernière robe, cette sublime chlamyde qu'elle portera au bal masqué, qui donne son nom au film).

12 Les éléments Art nouveau féminisent légèrement un univers auquel s'opposent les activités des deux personnages masculins férus de science et d'entomologie. Ceux-ci cherchent à s'emparer de l'espace en étalant à droite et à gauche leurs bestioles naturalisées. La lutte pour la conquête des espaces, lutte de sexes en somme, ne se fait pas attendre. Lorsque Pedro et Julián se préparent à déposer leurs boîtes d'insectes sur son guéridon préféré, Adela les réprimande énergiquement : «Vous ne placerez pas ici vos cafards répugnants! $»^{7}$ Les espaces se métamorphosant au fil des années, on arrive à l'acte II.

\section{Acte II. Temporalité fondée sur l'elliptique}

Vingt-cinq ans se sont écoulés. C'est la première grande ellipse. Techniquement traduite par les images des feuilles caduques en train de tomber d'un arbre du Retiro. C'est l'automne dans le parc et dans la vie des trois personnages qui approchent à présent de la cinquantaine. Dans le salon, l'espace matériel a légèrement changé. Quelques objets modern style ont disparu, d'autres demeurent. Leur absence ou leur présence participent à la réorganisation sémantique de l'espace et indiquent de facto que le temps est passé.

14 Cette deuxième partie s'ouvre sur un fondu enchaîné dévoilant la chambre d'Adela. Le rapprochement avec le plan précédent, celui du parc en automne, n'est donc pas dénué de sens. La caméra recule en travelling arrière et rentre dans la pièce à travers une fenêtre aux formes propres à l'Art nouveau, faisant ainsi irruption dans l'intimité féminine. La pièce est recadrée en plan rapproché. Adela nous tourne le dos mais on aperçoit son visage reflété dans le miroir dans lequel elle se regarde alors qu'elle fait sa toilette. La fenêtre est la même qu'en 1900. En revanche, le reste du mobilier, qui a évolué vers un style plus en concordance avec l'époque, a changé de même que la figure 
d'Adela qui a perdu de sa fraîcheur. Ainsi l'ameublement Art nouveau a-t-il laissé la place à des formes issues d'une conception rationaliste plus épurée et moins luxuriante. Seule la fenêtre parvient à faire le lien avec l'époque précédente. Alors que sur le visage d'Adela apparaissent des signes de vieillissement, des rides que le maquillage n'est plus à même de dissimuler, les lignes et les formes des meubles et des objets deviennent, quant à elles, plus lisses, dépourvues à présent de l'exubérance des principes constructifs modernistes. Cette mise à distance marque un contraste et accentue le désarroi que l'on perçoit chez Adela et dont on apprendra la cause à la fin de cet acte. Lorsque la voix off de Pedro lui annonce qu'une lettre de leur fille vient d'arriver, Adela lui refuse l'accès à la pièce (à savoir son espace, son intimité), se lève et la traverse afin de ramasser la lettre que son mari vient de glisser sous la porte. Ceci offre au spectateur une vue d'ensemble, presque impudique, d'un espace intime et pour ainsi dire secret.

Dans le salon, l'univers de Pedro et Julián a pris le dessus sur celui d'Adela. Au fur et à mesure qu'Adela a pris de l'âge, les boîtes d'insectes ont commencé à camper à leur aise. La science l'emporte sur la beauté plastique. Adela cède. Fatiguée physiquement et moralement, elle accepte mal sa décadence. Lorsque Pedro et Julián apprennent qu'elle est atteinte d'une maladie incurable, les deux amis se consacrent à la tâche de rendre heureuse la femme qu'ils aiment. Pour lui faire plaisir, ils jettent par la fenêtre les boîtes d'insectes, transformant à nouveau l'espace commun. Julián lui offre un bracelet en diamants et Pedro prépare un voyage qui ne sera pourtant jamais réalisé.

Adela se prépare, en effet, à entreprendre cet ultime voyage, le vrai, celui de la fin de la vie, pathos et thanatos ici réunis. Dans un beau plan d'ensemble filmé en contre-plongée, elle monte l'escalier moderniste qui lui permettait jusqu'alors l'accès à ses domaines, point de circulation entre des espaces à présent plus symboliques encore. Elle s'arrête sur le palier du premier étage et, s'adressant émue aux deux hommes de sa vie, leur fait ses adieux : "Merci pour votre amour $»^{8}$. Dépourvue de sa chlamyde, l'Adela-femme a accompli son cycle vital à l'instar des insectes collectionnés par les entomologistes. Il leur appartiendra, en bons scientifiques de la nature et des sentiments, de conserver sa beauté et sa mémoire, de la naturaliser pour ainsi dire. Fin du deuxième acte.

\section{Acte III. Un espace de substitution}

17 Même transition, même décor. Cette fois-ci, la caméra focalise en plan général la symbolique mortuaire des cyprès du Retiro. L'acte est bâti sur les principes de substitution et de réutilisation. Adelita, la petite fille presque adolescente de Pedro et Adela, s'est installée dans la maison de ses grands-parents vingt-cinq ans après la disparition de sa grand-mère. Elle apparaît d'emblée comme un personnage relevant du premier principe, la substitution. Celle-ci remplace la figure de la grand-mère auprès de Pedro et Julián, et ce, en vertu de la mise en place d'une identification : Adela et Adelita, dotées d'une ressemblance époustouflante (c'est la même comédienne, Conchita Montes, qui joue les deux rôles). Il y a donc une mort-renaissance du personnage de la femme aimée, de l'idéal féminin. Réincarnée dans la peau de sa petitefille Adelita, celle-ci rend possible une prolongation de la fiction grâce à une "insertion» du passé dans le présent diégétique. Mais ce leurre ne saurait se concrétiser véritablement sans le concours de certains objets qui affichent leurs fonctions polyvalentes. Adelita endosse le rôle d'Adela et assure la continuité au fur et à 
mesure qu'elle s'empare de l'univers matériel ayant appartenu à sa grand-mère à deux moments essentiels de sa vie, cinquante ou vingt-cinq ans auparavant. D'abord, c'est la chlamyde qu'elle portera dans un autre bal, puis le bracelet offert par Julián, peu avant que sa grand-mère ne s'éteigne. Ici intervient le principe de réutilisation. Les insectes, quant à eux, ont envahi à nouveau l'espace. Ils perdent encore une fois leur rôle de protagonistes dans la maison, mais non pas leur portée sémantique. Conservés au sec, les imagos représentent indirectement, mais plus fortement que jamais, ce désir de Pedro et de Julián de fixer une beauté éphémère dans le temps. En effet, l'imago est l'aboutissement du cycle de vie de l'insecte, une phase vouée à la reproduction qui ne dure que quelques heures ou quelques jours. Tout comme cela leur arrive avec le souvenir d'Adela. Devenus au fil des années les garants de la beauté et de la mémoire d'Adela, ils se doivent de les protéger coûte que coûte. C'est pour cette raison, pour préserver son amour et honorer sa mémoire, qu'ils renoncent à nouveau à leur passion et se prêtent à un petit jeu teinté de nostalgie innocente.

L'espace architectural se déconstruit et se reconstruit d'une façon surprenante aux yeux du spectateur et au sein même du récit. L'emplacement des objets devient par là même une donnée structurale. Leur ambivalence apparaît encore plus clairement lorsque Pedro et Julián s'imposent fiévreusement la tâche de récupérer dans le débarras les vieux meubles poussiéreux des années 1900, afin de recréer un espace appartenant à un passé révolu, celui où ils ont aimé la même femme avec une intensité presque identique. Le principe de réutilisation devient de plus en plus évident. En plan subjectif et statique, les regards des deux vieillards se heurtent à un champ plein mais vide dans le même temps. Ce qui est en jeu dans ce champ vide est l'expression de l'absence. L'image, en raison de son assimilation à un point de vue diégétique, offre une réponse plastique à une retrouvaille impossible, montre la tristesse des hommes et déclenche un processus de mémoire.

Le cycle vital se prolonge. Du point de vue diégétique, par la coprésence, dans cette dernière partie, de deux des trois âges de l'homme représentés : la vieillesse de Pedro et de Julián et la jeunesse d'Adelita. Du point de vue narratif, grâce à des procédés anaphoriques. La petite fille reproduit tout à la fin du film les derniers mots prononcés par sa grand-mère : «Merci pour votre amour ». Le récit est ainsi accompli. Neville a réussi, en ayant recours à la structure circulaire, à construire une topographie sentimentale et un hymne à la beauté entendue, tout comme dans la conception qu'Émile Gallé avait de l'Art, "au sens de Vérité »". Les différents parcours des personnages, ainsi que leurs rapports intimes avec les objets et les meubles, délimitent et décrivent l'espace architectural, construisent un véritable espace de la mémoire. Une mémoire des sentiments, où la vie s'incarne, se désincarne et se réincarne en un flux continu qui se perpétue au-delà de la mort. 


\section{NOTES}

1. Style dérivé du symbolisme français. Les objets se distinguaient par leur caractère spectaculaire, exquis et élégant, quoique toujours au service d'une fonctionnalité ornementale. Ils représentaient le triomphe d'un langage minoritaire et intellectualisé, canalisé jusque-là par la poésie, le théâtre ou la peinture, et étaient majoritairement destinés à un public bourgeois aux goûts raffinés. L'équivalent relatif en Espagne fut donc le Modernisme. Équivalent imparfait car le «Modernismo" ne supposait pas seulement la manifestation décorative de l'esthétique symboliste mais englobait également toutes les manifestations modernes apparues à la fin du siècle: naturalistes, impressionnistes, etc. Francesc Fontbona, "Art Nouveau. El estilo de la modernidad », Arte, nำ 70, Diciembre 2004, p. 84.

2. Émile Gallé, « Mobilier contemporain orné d'après la nature ", La Lorraine, Nancy, $1^{\mathrm{er}}$ janvier 1900, p. 23 et 24. Cité par Jean-Paul Midant, L'Art Nouveau en France, Paris, l'Aventurine, 1999, p. 123.

3. Ramón Rozas Domínguez, "Literatura y filmicidad en la obra de Edgar Neville: un caso inusual", Nickel Odeon, numéro 17, Invierno 1999. p. 196.

4. Valentín Berriochoa Sánchez-Moreno, "El Palacio de Longoria", R\&R, no 62, marzo 2002, p. 58-59.

5. "No dejes eso ahí encima, que se va a romper la mesita."

6. Klaus-Jürgen Sembach, L'Art Nouveau, Cologne, Taschen, 2002, p. 27.

7. “Aquí no me ponéis vuestras repugnantes cucarachas!”

8. "Gracias por vuestro amor."

9. Émile Gallé, "L'éducation professionnelle des ouvriers d'art », La Lorraine, Nancy, 1er janvier 1900, p. 22. Cité par Jean-Paul Midant, op. cit., p. 122.

\section{RÉSUMÉS}

En 1959, Edgar Neville mettait en scène un film dont le scénario fut écrit à partir d'une de ses pièces de théâtre, portant le même titre et écrite sept ans plus tôt. Cette source théâtrale détermine, non seulement la construction, la structure narrative et la conception des personnages du film, mais aussi la disposition des espaces et la fonction des objets qui dotent l'espace profilmique d'une signification objective et l'espace filmique d'une dimension émotionnelle et symbolique. Par ailleurs, ces derniers aspects revêtent une importance capitale pour ce qui est de la construction dramatique de l'œuvre. Neville arrive ainsi à édifier une sorte de topographie sentimentale, dans laquelle, les divers recours des personnages, de même que les relations particulières qu'ils entretiennent avec objets et meubles - qui remplissent des fonctions polyvalentes durant toute la narration - délimitent et décrivent l'espace architectonique et construisent un espace authentique de la mémoire.

En 1959, Edgar Neville dirige un film cuyo guión fue escrito a partir de la obra teatral del mismo título que el mismo autor escribiera siete años antes. Este origen teatral determina no solamente la planificación, la estructura narrativa y la concepción de los personajes de la película, sino también la disposición de los espacios y la función asumida por los objetos, que dotan al espacio 
profílmico de una significación objetiva y al espacio fílmico de una dimensión emocional y simbólica. Estos últimos aspectos, por otra parte, revisten capital importancia en lo tocante a la construcción dramática de la obra. Neville consigue de esta manera edificar una suerte de topografía sentimental en la que los diferentes recorridos de los personajes, al igual que las particulares relaciones que éstos establecen con los objetos y con los muebles - que desempeñan funciones polivalentes durante toda la narración - delimitan y describen el espacio arquitectónico y construyen un genuino espacio de la memoria.

\section{INDEX}

Mots-clés : espace urbain, cinema, Neville, El baile

Palabras claves : espacio urbano, cinema, Neville, El baile

\section{AUTEUR}

\section{ALFONSO SAN MIGUEL MONTES}

Université de la Sorbonne/Paris IV, France 